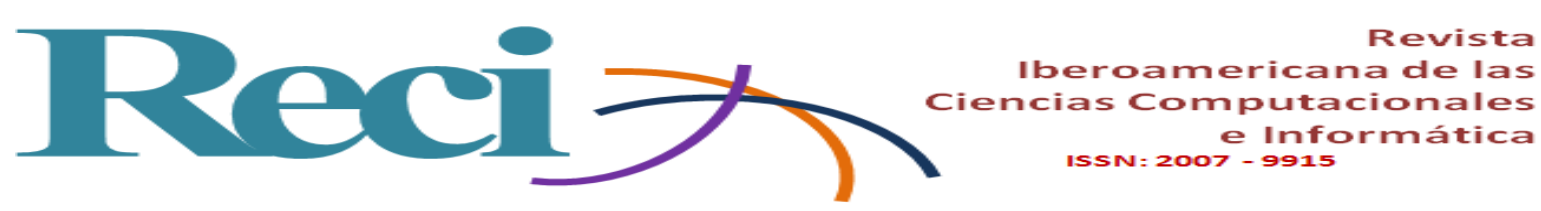

https://doi.org/10.23913/reci.v10i19.103

Artículos científicos

\title{
Automatización de los procesos de gestión de proyectos de investigación: caso ITSC
}

Automation of Research Project Management Processes: ITSC Case

Automação de processos de gestão de projetos de pesquisa: caso ITSC

Ever Alcudia Fuentes

Instituto Tecnológico Superior de Comalcalco, México

ever.alcudia@comalcalco.tecnm.mx

https://orcid.org/0000-0001-6432-7967

Elías Rodríguez Rodríguez

Instituto Tecnológico Superior de Comalcalco, México

elias.rodriguez@comalcalco.tecnm.mx

https://orcid.org/0000-0002-1012-8169

David Ramírez Peralta

Instituto Tecnológico Superior de Comalcalco, México david.ramirez@comalcalco.tecnm.mx https://orcid.org/0000-0003-3181-1351

Alejandro López Jiménez

Instituto Tecnológico Superior de Comalcalco, México alejandro.jimenez@comalcalco.tecnm.mx https://orcid.org/0000-0002-2042-6975 


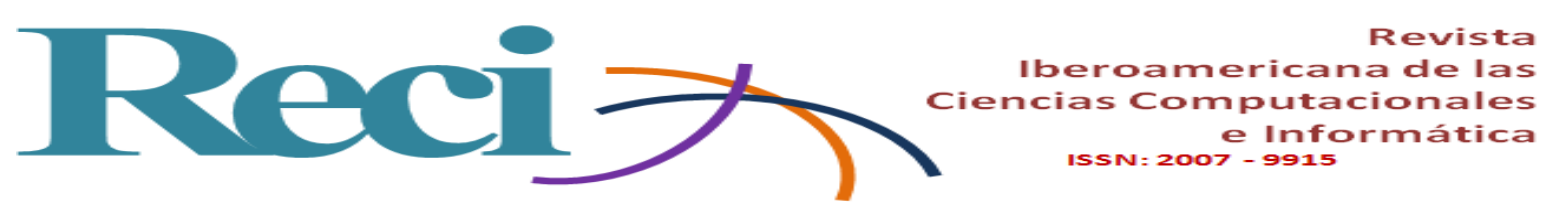

\section{Resumo}

Este trabalho tem como objetivo desenvolver um sistema de informação para gerir e automatizar o acompanhamento de projetos de investigação no Instituto Tecnológico Superior de Comalcalco (ITSC). Foi aplicada uma metodologia de tipo misto, caracterizada pela sua abordagem interdisciplinar. Os dados foram obtidos por meio de entrevista virtual com cada um dos envolvidos no processo de pesquisa do ITSC. A amostra total foi de 30 pesquisadores. A contribuição deste projeto é a automação do processo de registro e acompanhamento dos projetos de pesquisa dos docentes pesquisadores do ITSC. Para o desenvolvimento do software foram atendidas as necessidades básicas de gestão de projetos (geração de relatórios por períodos, cadastro e exportação em formato CSV ou PDF e até envio para impressão, bem como o protocolo dos projetos de pesquisa em execução e no desenvolvimento do currículo, graças ao qual o desempenho dos pesquisadores pode ser medido). É uma importante ferramenta que contribui para reduzir o tempo gasto na busca de documentação, o consumo de papéis, facilitando o acompanhamento, a busca e o armazenamento dos projetos, além de conhecer em tempo real o andamento dos projetos de pesquisa que estão sendo desenvolvidos pelo professores.

Palavras-chave: automação, gestão, projetos de pesquisa.

Fecha Recepción: Julio 2020

Fecha Aceptación: Diciembre 2020

\section{Introducción}

En el Instituto Tecnológico Superior de Comalcalco (ITSC), los procesos académicos y administrativos se solían llevar a cabo de forma analógica. En el año 2009, sin embargo, se implementó el sistema de información llamado Sistema de Seguimiento Académico (SSA). Esta herramienta tecnológica cuenta con los módulos Inicio, Escolares, Reportes, Utilerías y Cierre de sesión, pero no contempla el proceso de seguimiento de proyectos de investigación, ya que esta actividad no se consideró en su momento como primordial.

A raíz del crecimiento de la matrícula de estudiantes y la creación de nuevas carreras en el ITSC, se creó el Departamento de Investigación y Posgrado. Encargado de fomentar la investigación, ha contribuido al desarrollo de proyectos académicos. El registro de este tipo de proyectos ha incrementado a tal grado que ha surgido la necesidad de contar con más herramientas de seguimiento y mejora continua de los productos generados, tales como un proceso de registro más eficiente, la participación en ponencias, la publicación de artículos, conseguir más perfiles deseables, participación de estudiantes con tesis y vinculación con el sector empresarial y gubernamental. 


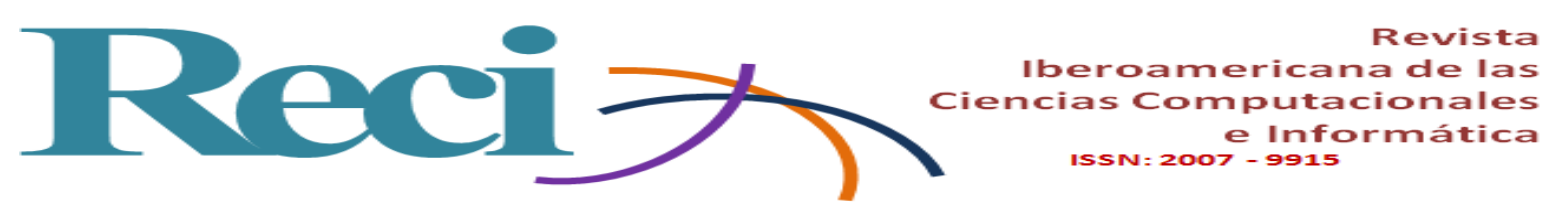

De ahí la importancia de mejorar el control de los procesos para la administración de proyectos de investigación en el ITSC. Actualmente, estos se registran en un documento de Microsoft Word y son almacenados dentro de carpetas en el equipo de cómputo del encargado del Departamento de Investigación y Posgrado, de acuerdo con el periodo escolar en el que se registra cada proyecto, con una duración de un año, es decir, 12 meses.

El resultado de este estudio sobre el desarrollo de proyectos de investigación evidencia que $60.6 \%$ de los profesores y alumnos no está desarrollando o se encuentra inscrito en algún proyecto de investigación; mientras que el porcentaje restante, $39.4 \%$, sí está trabajando al menos uno. Esto se relaciona con el aumento paulatino de profesores y alumnos cada vez más interesados en esta actividad en el ITSC.

La investigación científica, a través del desarrollo de proyectos de investigación, tiene la noble función de fomentar y acrecentar el conocimiento a través del pensamiento crítico. Actualmente, se trata de uno de los indicadores más importantes para todas las instituciones de educación superior en México, y en particular para el ITSC. La investigación desarrollada en estas instituciones es observada tanto por diferentes organismos públicos como privados. Son una fuente de financiamiento, capaces de proveer bienestar académico a las organizaciones.

En efecto, la investigación es una actividad sustantiva del quehacer académico en el ITSC: aumentar el caudal de conocimiento sobre un tema en particular que busca contribuir al desarrollo del entorno. A través del análisis de los datos, se descubrió que la mayoría de los investigadores y alumnos prefiere realizar proyectos de investigación en el área de desarrollo tecnológico. Desafortunadamente, este interés no es recíproco, ya que la comunidad tecnológica no está muy involucrada en las ciencias de la educación.

El Departamento de Investigación y Posgrado comenzó a detectar nuevas problemáticas con la administración de los proyectos de investigación que ahí se desarrollan. Sin duda es necesario llevar a cabo un trabajo sistemático sobre los procesos y productos de investigación tecnológica y así coadyuvar con la vinculación de los diferentes sectores económicos y sociales. No contar con un debido control de estos es un foco rojo que debe ser atendido cuanto antes. De ahí la valía de automatizar el proceso de registro y lograr que el seguimiento de los proyectos de investigación involucre a todas las partes (profesores, alumnos, áreas administrativas, sectores productivos), al igual que facilitar el acceso a la información de cada uno de los proyectos mediante la creación de informes y estadísticas. 


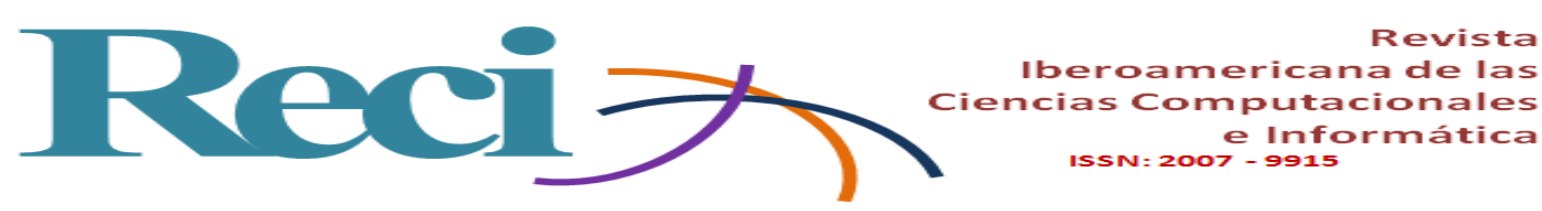

\section{Materiales y métodos}

De acuerdo con Hernández y Mendoza (2008), la metodología de investigación mixta se caracteriza por su enfoque interdisciplinario y suele ser utilizada para propósitos aplicados y la construcción de teorías.

Los datos fueron obtenidos mediante la aplicación de una entrevista virtual a cada uno de los involucrados en el proceso de investigación en el ITSC. Se entrevistó a una muestra total de 30 investigadores, quienes contribuyeron en gran parte para el levantamiento de la información.

El volcado de los datos se realizó mediante un análisis interpretativo. Se examinó minuciosamente la naturaleza de las respuestas, tomando en cuenta el contexto en el que cada uno de los entrevistados se desenvuelve. Como resultado de este análisis, se detectó la necesidad de desarrollar un sistema de información basado en tecnologías web para la automatización de los procesos de gestión de proyectos de investigación en el Departamento de Investigación y Posgrado del ITSC.

La pregunta que guio esta investigación fue la siguiente: ¿cómo el desarrollo de un sistema de información automatizado mejora los procesos de gestión de proyectos de investigación en el ITSC?

El proyecto se aplicará únicamente en el Departamento de Investigación y Posgrado del ITSC y abarcará los procesos de registros, el seguimiento de los proyectos de investigación y la generación de informes.

Igualmente, a partir del análisis efectuado en este trabajo de investigación, se detectaron algunas limitaciones: la falta de recursos económicos para la implementación del software, la duración del tiempo de desarrollo del proyecto y situaciones de variables no controlables como el estado de salud de los participantes o sucesos de fuerzas naturales.

Actualmente, en el ITSC el proceso de registro y seguimiento de los proyectos de investigación se realiza a través de Microsoft Word. Y cuando se requiere conocer el grado de avance de los proyectos de investigación, los profesores investigadores y alumnos involucrados tienen que solicitarlo directamente al Departamento de Investigación y Posgrado. Algo importante en este ámbito es identificar a los profesores con proyectos de investigación para asignar en la carga académica, horas de investigación correspondiente, así como la hoja de vida, que es mostrar toda la información de profesor investigador relacionada con investigaciones y su desempeño laboral.

El objetivo, pues, es desarrollar un sistema de información para controlar la administración de proyectos de investigación que automatice el seguimiento de los proyectos, entrega de reportes, organice la información personal, laboral y profesional de los profesores 


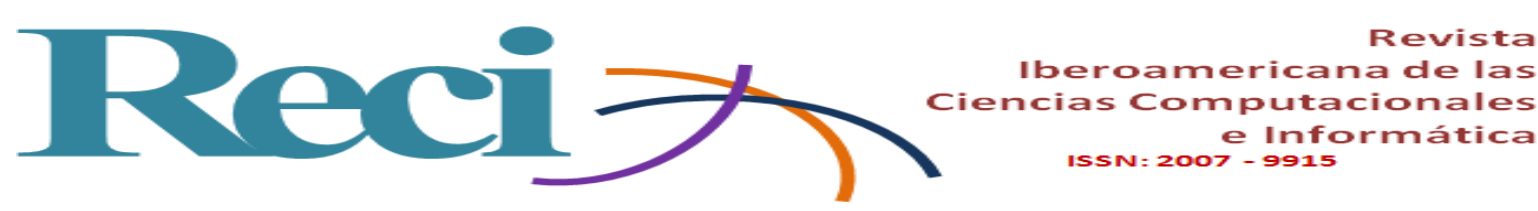

investigadores del ITSC a través de la hoja de vida. Este sistema será una herramienta importante para la institución; permitirá minimizar el tiempo de búsqueda de documentos, reducir el consumo de papel y liberar el espacio físico donde se ubica actualmente la información.

\section{Recolección de datos}

Para el levantamiento de datos, se determinó la aplicación de una entrevista presencial y una encuesta virtual a los involucrados en el proceso de investigación en el ITSC. En total se entrevistó a 30 docentes investigadores y a la jefa de departamento en cuestión. Asimismo, a una muestra de 93 personas, quienes contribuyeron en la fase del levantamiento de información. A continuación, se presenta un reporte de los resultados obtenidos.

\section{Análisis de la información recolectada mediante entrevista}

El análisis de datos se realizó de acuerdo con el método de comparación constante, adaptado y descrito por Maykut y Morehouse (1994). La principal fuente de información para el análisis de datos corresponde a las entrevistas realizadas.

La mayoría de los entrevistados (95\%) cree que, para generar ideas de proyectos, deben identificar la problemática, proponer una solución a esta, redactar objetivos, alcances, limitaciones, justificación y cronograma de actividades, esto a partir de un análisis de la realidad del entorno, así como tomando en cuenta la agenda estatal de desarrollo De la misma forma, se menciona seguir el uso de formatos que contienen los puntos necesarios para documentar un proyecto, al igual que el protocolo institucional; algunos cuantos sugieren la estructura que maneja la Universidad Nacional Autónoma de México (UNAM) y el Instituto Politécnico Nacional (IPN) y la metodología de investigación establecida por Roberto Hernández Sampieri.

\section{Análisis de la información recolectada mediante encuesta}

Para el levantamiento de datos cuantitativos, la herramienta utilizada fue la encuesta, la cual se aplicó de forma virtual, haciendo uso de Google Forms. Esta fue realizada a una muestra de 93 individuos. A continuación, se hace una representación gráfica de los datos obtenidos.

Como se muestra en la tabla 1, 40.9\% de los encuestados sí está desarrollando o está involucrado en un proyecto de investigación, mientras que $59.1 \%$ respondió que no realizan actualmente un proyecto de investigación. A pesar de que una de las funciones de las instituciones de educación superior es desarrollar investigación, se observa que un alto porcentaje de alumnos y profesores no está llevando a cabo dicha función. 


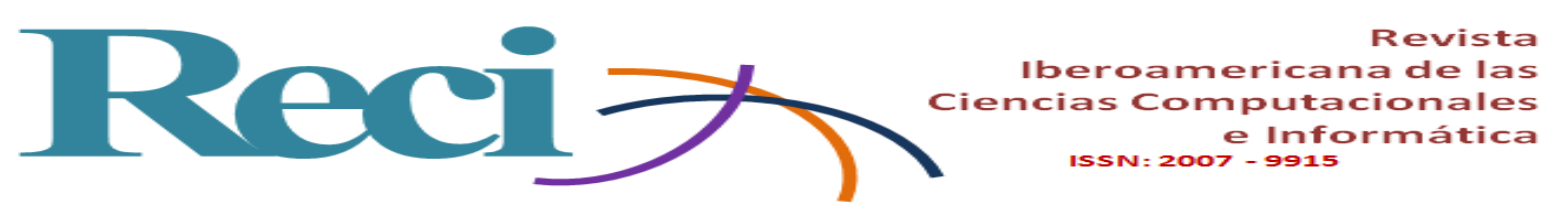

Tabla 1. Tabulación de datos sobre proyectos de investigación

\begin{tabular}{|l|l|}
\hline \multicolumn{2}{|c|}{ ¿Desarrollas actualmente algún proyecto de investigación en el ITSC? } \\
\hline Sí & $40.9 \%$ \\
\hline No & $59.1 \%$ \\
\hline
\end{tabular}

Fuente: Elaboración propia

Respecto a la pregunta “¿Tipos de proyectos de investigación?, los proyectos tecnológicos acumulan el mayor porcentaje con $34.7 \%$, siguiendo la investigación básica con $23.3 \%$, innovación con $16.7 \%$, servicios científicos y tecnológicos con $8.3 \%$, ciencias de la tierra y medio ambiente con $6.8 \%$, ciencias económico-sociales con $5.5 \%$, ciencias naturales con $2.7 \%$, ciencias de la educación con $1.4 \%$ y, por último, ciencias bioquímicas con $0.6 \%$. Así pues, el área en la que más se enfocan es el desarrollo tecnológico y en la que menos, las ciencias bioquímicas.

Tabla 2. Tabulación de datos sobre tipos de proyectos

\begin{tabular}{|l|l|}
\hline \multicolumn{2}{|c|}{ ¿Tipos de proyectos de investigación? } \\
\hline Desarrollo Tecnológico & $34.7 \%$ \\
\hline Innovación & $16.7 \%$ \\
\hline Ciencias naturales & $2.7 \%$ \\
\hline Ciencias económico-sociales & $5.5 \%$ \\
\hline Ciencias bioquímicas & $0.6 \%$ \\
\hline Ciencias de la tierra y del medio ambiente & $6.8 \%$ \\
\hline Ciencias de la educación & $1.4 \%$ \\
\hline Investigación básica & $23.3 \%$ \\
\hline Servicios científicos y tecnológicos & $8.3 \%$ \\
\hline
\end{tabular}

Fuente: Elaboración propia

\section{Resultados}

Lo conseguido en esta investigación genera un impacto directo en el área de investigación y posgrado del ITSC. Esta área se compone por un total de 50 docentes investigadores, quienes trabajan con una cantidad importante de alumnos colaboradores en proyectos de investigación (30\%).

Ahora bien, según $90 \%$ de los docentes entrevistados, las características de un proyecto de investigación deben ser la siguientes: viabilidad del proyecto, que resuelva una problemática real, que sea un proyecto de innovación, que la solución sea de acuerdo con la línea de 


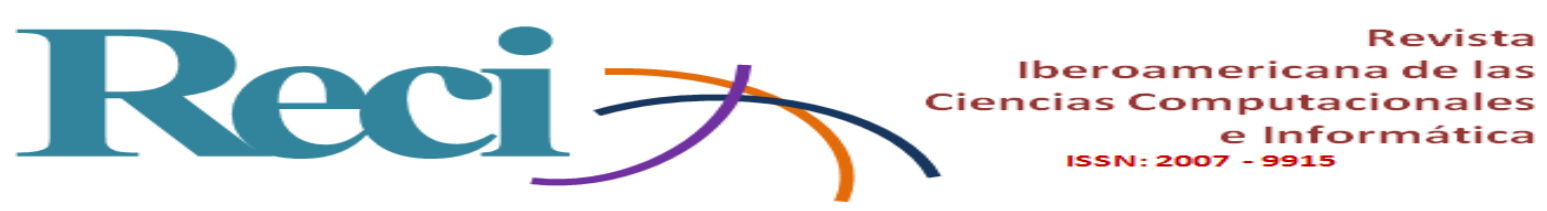

\section{Discusión}

La necesidad de contar con sistemas de información que permitan registrar, controlar y llevar el seguimiento de proyectos ha provocado que las instituciones educativas desarrollen iniciativas propias que permitan mejorar y facilitar este proceso, sin embargo, también existen sistemas de información en línea de empresas y organizaciones privadas que satisfacen dichas necesidades.

Por ejemplo, el software de gestión empresarial Scoro ayuda a gestionar proyectos empresariales. Cuenta con los módulos de gestión de proyecto, gestión de trabajo, ventas, finanzas, informes y paneles; presenta planificación, programación y seguimiento de proyectos; ofrece la posibilidad de administrar todos los proyectos en vistas personalizables y visualizar el progreso utilizando la línea de tiempo del proyecto. También prioriza las asignaciones, evita superposiciones y ayuda a establecer plazos realistas. Por último, incluye calendarios, listas de tareas, hojas de tiempo y un rastreador de tiempo integrado. Su gran desventaja radica en el costo, ya que muchas organizaciones educativas no cuentan con presupuesto para este tipo de necesidades, por lo que, como ya se mencionó, optan por desarrollar sus propios sistemas de información.

Los sistemas online Trello, Write y Basecamp permiten que los equipos trabajen de forma más colaborativa y sean más productivos. Incluye tarjetas, listas y tableros que ayudan a que los equipos se organicen y prioricen sus proyectos de forma divertida, flexible y provechosa. Sin embargo, estos softwares no consideran como gratuito la opción de guardar por tiempo indefinido la información, por lo que, si se desea este servicio, habría que pagar.

El software resultante de esta investigación se desarrolló de acuerdo con las necesidades del Departamento de Investigación y Posgrado del ITSC. Los módulos de principal interés son los que ayudan a la gestión de los proyectos, la hoja de vida y los reportes periódicos. Además, se desarrollaron tres perfiles pensados para cada tipo de usuario (estudiantes, profesores/investigadores y administrativos). Cada versión cuenta con módulos específicos para que el usuario realice las actividades de acuerdo con el rol que tendrá en los proyectos a desarrollar. 


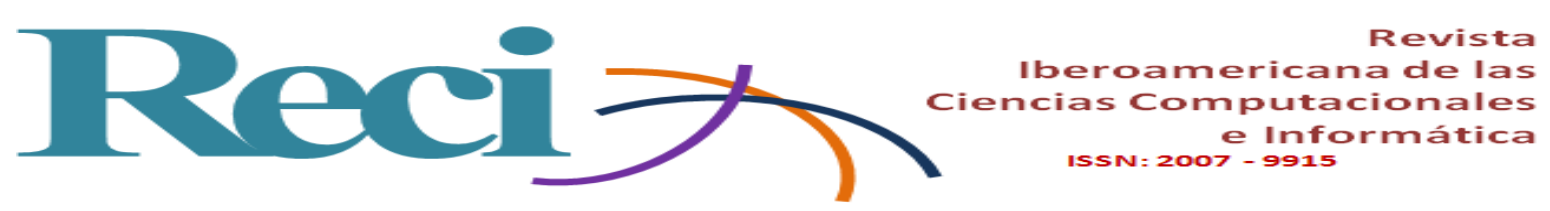

\section{Referencias}

Chaviano, Y. y Hernández, A. (2006). Herramientas automatizadas para la gestión de proyectos. Ingeniería Industrial, 37(2-3), 67-74. Recuperado de https://n9.cl/he1d2.

Fuentes, L. (2018). The Future of Cities is Smart, Inclusive and Sustainable: Research and Proposal of Smart City Layer Implementation for Mexico. RIIIT. Revista Internacional de Investigación e Innovación Tecnológica, 6(31). Recuperado de https://n9.cl/csi1o.

García, J., Minero, J. J., Muñoz, M. A. y Mejía, J. (2015). Mejora de procesos para la administración de proyectos en instituciones de nivel superior. Revista Electrónica de Computación, Informática, Biomédica y Electrónica, (1). Recuperado de https://n9.cl/46ifu.

Hasan, S., Román, P. A., Piñero, P. Y., Sadeq, G. y Leyva, M. (2019). Sistema de apoyo a la toma de decisiones basado en mapas cognitivos neutrosóficos para instituciones que atienden a embarazos con alto riesgo por enfermedades cardiovasculares. Revista Cubana de Ciencias Informáticas, 13(4), 16-29. Recuperado de https://n9.cl/stnhs.

Hernández, R. y Mendoza, C. (2018). Metodología de la investigación. Las rutas cuantitativa, cualitativa y mixta. Ciudad de México, México: Editorial McGraw-Hill.

Lerma, I. y Bauer, J. R. (2018). Características de un sistema de información para la asignación y gestión de apoyos a proyectos gubernamentales. Agroproductividad, 11(3), 141-147. Recuperado de https://n9.cl/z9kd0

Linares, J. A. y Geizzelez, M. L. (2007). Administración de proyectos en ingeniería del software. Telos, 9(1), 26-41. Recuperado de https://n9.cl/4vwz0.

López, C., Sánchez, Á., Pardo, R. D. y Aristizábal, S. (2016). Modelo de gestión de proyectos de la Universidad Eafit aplicados al sistema general de regalías en ciencia, tecnología e innovación. Revista Ciencias Estratégicas, 24(36), 271-289. DOI

Mar, O., Zulueta, Y., del Rosario, M. y Leyva, M. (2015). Motor de inferencia decisional en sistema informático para la evaluación del desempeño. Revista Cubana de Ciencias Informáticas, 9(4), 16-29. Recuperado de https://n9.cl/lsqc.

Martínez, M., García, I., Figueroa, E. y Fernández, A. (2018). Herramienta de evaluación de la propiedad intelectual para proyectos: estudio de caso en proyectos de ciencia, Tecnología e innovación. Palabra Clave, 8(1). Recuperado de https://doi.org/10.24215/18539912e061.

Maykut, P. and Morehouse, R. (1994) Beginning Qualitative Research. A Philosophical and Practical Guide. London, England: The Falmer Press. 


\begin{tabular}{|c|c|}
\hline & 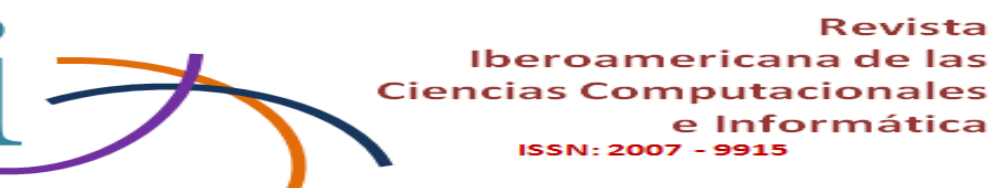 \\
\hline Rol de Contribución & Autor (es) \\
\hline Conceptualización & Ever \\
\hline Metodología & Ever \\
\hline Software & Elias \\
\hline Validación & Alejandro \\
\hline Análisis Formal & David \\
\hline Investigación & Ever, Elias, David, Alejandro. <<igual>> \\
\hline Recursos & Elias. \\
\hline Curación de datos & David \\
\hline $\begin{array}{l}\text { Escritura - Preparación del } \\
\text { borrador original }\end{array}$ & Alejandro \\
\hline $\begin{array}{l}\text { Escritura - Revisión y } \\
\text { edición }\end{array}$ & Ever, Elías, David, Alejandro. . <<igual >> \\
\hline Visualización & David, Alejandro . <<igual >> \\
\hline Supervisión & David, Alejandro . <<igual >> \\
\hline Administración de Proyectos & Elias, Ever . <<igual >> \\
\hline Adquisición de fondos & Ever \\
\hline
\end{tabular}

